\title{
Article \\ Portable Near-Infrared Spectroscopy as a Screening Test of Corrosive Solutions Concealed in Plastic Containers
}

\author{
Alvaro Varela Morillas (D) and Nunzianda Frascione * \\ Department of Analytical, Environmental and Forensic Sciences, King's College London, 150 Stamford Street, \\ London SE1 9NH, UK; alvaro.varela_morillas@kcl.ac.uk \\ * Correspondence: nunzianda.frascione@kcl.ac.uk; Tel.: +44-(0)20-7848-4462
}

Citation: Varela Morillas, A.; Frascione, N. Portable Near-Infrared Spectroscopy as a Screening Test of Corrosive Solutions Concealed in Plastic Containers. Appl. Sci. 2022, 12, 2770. https://doi.org/10.3390/ app12062770

Academic Editor: Alessandra Biancolillo

Received: 24 January 2022

Accepted: 4 March 2022

Published: 8 March 2022

Publisher's Note: MDPI stays neutral with regard to jurisdictional claims in published maps and institutional affiliations.

Copyright: (c) 2022 by the authors. Licensee MDPI, Basel, Switzerland. This article is an open access article distributed under the terms and conditions of the Creative Commons Attribution (CC BY) license (https:/ / creativecommons.org/licenses/by/ $4.0 /)$.
Featured Application: Analysis of inorganic acids and bases through closed plastic containers.

\begin{abstract}
The use of corrosive substances for criminal intent has recently increased in many countries, with 619 violent assaults recorded from 2019 to 2020 only in the UK. Criminals often conceal corrosive solutions, such as common household cleaners, in inconspicuous plastic bottles and splashing the content in order to incapacitate a victim while committing a robbery or to cause physical harm. There is currently no method available to law enforcement for the safe identification of these corrosive substances without being exposed to them. In this work, the feasibility of a near infrared (NIR) handheld spectrometer for the screening of corrosive inorganic solutions through plastic bottles is investigated. First, a training set comprising samples of five different corrosives was used to build a spectral library for data analysis and chemometric model design. Four models were then tested on three hundred samples of corrosive substances, as well as harmless substances such as water and soft drinks, to evaluate their performance. The models designed identified the corrosive substances in scenarios of concentrated solutions, showcasing the potential capability of this technique for the pre-screening of corrosive substances.
\end{abstract}

Keywords: near infrared; spectroscopy; sulfuric acid; sodium hydroxide; corrosive; acid attack

\section{Introduction}

Recent reports in the UK have shown an increasing trend in the use of corrosive substances for different criminal activities. A survey from 2021 revealed that, between 2015 and 2017, 1187 criminal offences involving corrosive substances had been reported by eight police forces in England and Wales [1]. In addition, data collected by the Home Office as part of the 'Serious Violence Strategy' showed that "between April 2019 and March 2020, 619 violence against the person and robbery offences that involved a corrosive substance had been recorded by the police in England and Wales (excluding Greater Manchester Police)" [2]. These reports also highlighted that violence against the person was the predominant type, making up to $77 \%$ of the total offences [1]. This type of crime is often carried out by an individual carrying the corrosive substance in an inconspicuous plastic container (e.g., a plastic bottle), and throwing the liquid on the victim with the intention to cause injury. Considering that only $8 \%$ of the victims of these offences avoid injuries and that $35 \%$ of the products used are readily available household products [1], local and national authorities have started to evaluate measures for preventing such crimes. Strategies to limit the access to these substances in retail stores, as well as additional regulations concerning these substances in the national legislation-e.g., the Offensive Weapons Act 2019 in the UK-have been introduced. Despite this, consumers can still easily access corrosive products that are sold as common household products. Therefore, there is an urgent need to develop strategies for the on-site detection and analysis of these substances through the selection and testing of analytical techniques that can be readily used by a law enforcement on the street. 
A method that could prove useful for this purpose is Near Infrared spectroscopy (NIR)-based analysis. NIR is a technique of great value for analytical screening, mainly due to its speed and the fact that it requires no sample preparation [3]. Furthermore, the capability of NIR to analyse samples through glass and plastic containers [4-7] is a desirable feature, particularly in scenarios where the sample to be analysed can be harmful if handled in an open container. Finally, the affordability and relative simplicity of NIR instruments have allowed the design and use of portable and handheld devices for the qualitative and quantitative analysis of a wide range of substances that have already being explored, from quality analysis in the food industry, soil analysis and even for forensic purposes [8-11]. Based on this, the present study focused on evaluating the applicability of portable NIR, as an on-field test of corrosive substances.

In addition to this, previous research has explored the combined use of NIR with chemometric modelling as a powerful tool for the analysis and discrimination of unknown samples $[8,9,12]$. Interpretation of raw NIR spectral data is complex and cumbersome without the aid of software and mathematical processing. Methods such as mean-centering, standard normal variate (SNV) or first and second derivative have allowed a better understanding of the information provided by the broad peaks of NIR spectra [13-15]. These methods allow the user to remove unnecessary information, noise and uncontrolled variations of the baseline, or separate overlapping peaks. Additionally, these pre-treatment methods are often combined with multivariate data analysis methods such as Partial Least Square Regression (PLSR) [16], Soft Independent Modelling of Class Analogies (SIMCA) [17] and Principal Component Analysis (PCA) [18] in order to identify the main sources of variability among the classes defined by the user and, in turn, assist in the clustering of new unknown samples. These approaches have become a standard practice when using NIR for analytical purposes, particularly in the design of supervised methods, in different scientific fields $[8,9,12,19-22]$.

$\mathrm{SCiO}^{\mathrm{TM}}$ is a handheld NIR spectrometer designed and manufactured by Consumer Physics Inc. (Tel Aviv, Israel). The device, controlled via Bluetooth from a mobile device such as a smartphone, can scan samples in the region between 740 and $1070 \mathrm{~nm}$, also known as the region of the third overtone in the NIR spectrum [3], in diffuse reflectance mode. Once samples have been scanned, a software allows the user to build a training spectral library that can be processed and analysed through machine learning methods to develop a chemometric model tailored to the final analysis the user aims to perform. These models can then be implemented in a mobile app so the end user can easily and quickly visualise the results of a new scan on their mobile device. This instrument has already been tested in a wide range of scientific fields [23-27] including the field of forensic science [8].

In this study, five corrosive substances (commonly used in corrosives attacks) have been selected and analysed. These corrosive substances have been scanned through commercially available bottles made of Polyethylene terephthalate (PET) and High-Density Polyethylene (HDPE) using $\mathrm{SCiO}^{\mathrm{TM}}$ to assess whether these substances can be correctly identified through the container walls, therefore preventing any exposure to the corrosive in question. Furthermore, the methodology explored during this study was restricted exclusively to the tools available within the $\mathrm{SCiO}^{\mathrm{TM}}$ and its associated software to assess the potential implementation of such commercial instruments among the intended end users, e.g., police forces. This is, to the best of the authors' knowledge, the first study to assess the use of NIR, and specifically portable NIR, for the qualitative analysis of corrosive substances in criminal investigations.

\section{Materials and Methods}

\subsection{Corrosives}

Concentrated sulfuric acid $\left(\mathrm{H}_{2} \mathrm{SO}_{4}\right) 97 \%$, hydrochloric acid $(\mathrm{HCl}) 37 \%$, sodium hypochlorite $\left(\mathrm{NaHClO}_{3}\right) 10-15 \%$ and ammonium hydroxide $\left(\mathrm{NH}_{4} \mathrm{OH}\right) 30-33 \%$ from Scientific Laboratory Supplies Ltd. (SLS) (Nottingham, UK), as well as sodium hydroxide ( $\mathrm{NaOH}) 50 \%$ from Fisher Scientific Ltd. (Loughborough, UK) were purchased as stock solutions. These were 
then diluted in water to match concentrations reported in the Offensive Weapons Act 2019 (Schedule 1), that is, the concentration limit that can be legally sold in retail stores. Some of these solutions (i.e., sulfuric acid and sodium hypochlorite), were further diluted due to the PET bottles not being stable to hold the stock solution for long periods of time. The working concentrations used in this study are listed in Table 1.

Table 1. Working concentrations of the corrosive aqueous solutions explored as analytical targets.

\begin{tabular}{cccc}
\hline Corrosive & Stock Solution & Concentration 1 & Concentration 2 \\
\hline Sulfuric acid & $97 \%$ & $30 \%$ & $15 \%$ \\
Ammonium & $30-33 \%$ & $10 \%$ & - \\
hydroxide & $37 \%$ & $10 \%$ & - \\
Hydrochloric acid & $50 \%$ & $12 \%$ & - \\
Sodium hydroxide & $10-15 \%$ & $4 \%$ & $1 \%$ \\
Sodium hypochlorite & & & \\
\hline
\end{tabular}

\subsection{Containers}

Reportedly, the corrosive substances used to attack individuals are concealed in commercially available plastic bottles often with sports caps. For the purpose of mimicking real case scenarios, the different corrosive substances analysed in this study were stored in PET bottles (Lucozade Sport $500 \mathrm{~mL}$ and Fruit Shoots $275 \mathrm{~mL}$, both available with sports caps). Two different types of PET bottles were chosen to ensure that both transparent (i.e., Lucozade) and coloured (i.e., Fruit Shoots) PET were included to assess whether the colour of the plastic has an influence on the performance of the analysis. In addition to PET, HDPE bottles (Ampulla Ltd., Hyde, UK, $500 \mathrm{~mL}$ ) were also included. Despite HDPE not being common in the soft-drink industry, its high compatibility with most corrosive substances and its presence in cosmetic product containers make this material of potential interest for the purpose of this study.

\subsection{Instrument}

The $\mathrm{SCiO}^{\mathrm{TM}}$ molecular sensor (v1.5, Consumer Physics Inc., Tel-Aviv, Israel) was used to gather spectral data of corrosive solutions in different plastic containers in the wavelength range of 740 to $1070 \mathrm{~nm}$ in diffuse reflectance mode. The devices wavelength resolution is $<10 \mathrm{~cm}^{-1}$ and the sampling interval is $1 \mathrm{~nm}$. The device was fully controlled via Bluetooth from a smartphone.

\subsection{Sample Preparation and Data Collection}

In order to build a data library that could train the instrument to correctly identify the different corrosive substances, the available samples were divided into two groups, training and testing set, in the proportions 60:40 (as represented in Figure 1) to ensure proper validation could be performed [28].

For each combination of corrosive substance/container type and concentration, 3 sets of 5 samples were prepared by pouring $100 \mathrm{~mL}$ of the solution into 5 plastic bottles of each plastic material. Before analysis, the bottles were left to settle for $10 \mathrm{~min}$ to assess any potential damage to the container by the solution. After ensuring that the solution was compatible with the plastic material, each sample was scanned by holding the instrument on the side, approximately $5 \mathrm{~mL}$ away from the bottle. This was repeated 10 times. The procedure has been illustrated in Figure 2.

After the spectra from the training set were collected, the bottles with corrosive solutions were kept under conditions of room temperature and lighting for a period of time no longer than $8 \mathrm{~h}$ to observe any potential visible changes to the plastic materials in contact with the corrosive substance. Examples of the raw spectra gathered at this stage can be found in Figure 3. Since this study exclusively explored the capabilities of the commercial tools associated with the device used, only the raw spectra could be plotted at this stage. 


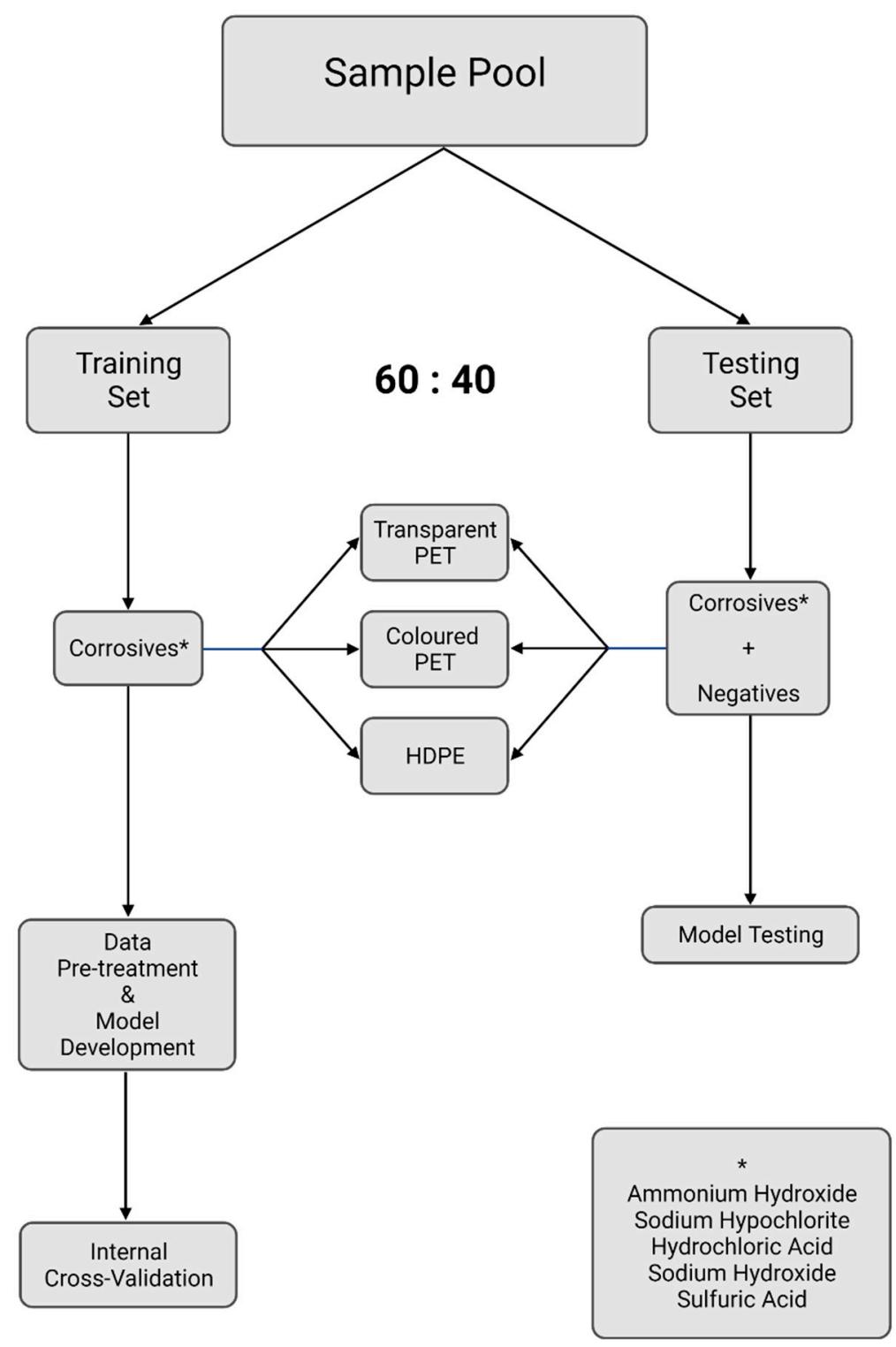

Figure 1. Experimental workflow from sample collection to model performance evaluation. Created with BioRender.com. * presents corrosive compounds.

\subsection{Data Analysis}

Analysis of the training set spectral data was performed using Consumer Physics Inc. software, the $\mathrm{SCiO}^{\mathrm{TM}} \mathrm{Lab}$. This software provides the user with a small range of pre-processing methods that can be applied to the data within the library. The output or data gathered with the studied instrument is made up of a reflectance spectrum comprised of a total of 300 discrete reflectance points in a one-nanometre scale that ranges from 740 to $1040 \mathrm{~nm}$. In order to eliminate any noise from the environment and the instrument, as well as to ensure the spectra were reproducible, a series of pre-processing techniques commonly used in the field of chemometrics, and included in the instrument's software, were applied to the data. A combination of Subtract Average, Standard Normal Variate (SNV), 1st Derivative, with a window of 35 points and polynomial degree of 2, and wavelength selection of the spectral region between 800 to $1000 \mathrm{~nm}$, was identified as the best preprocessing method and used to design a series of models with different parameters using the Partial Least Squares (PLS) algorithm [29,30] employed by the instrument's software. Their theoretical performance was assessed through internal 'leave-one-out' (LOO) crossvalidation performed automatically by the software once a model was built. This cross- 
validation follows the venetian blinds approach where the samples within the training library are split into cross-validation groups and then samples within those groups are chosen in an alternating fashion, removed from the calibration group and tested against it to perform the cross-validation. In the particular case of the LOO validation, the number of cross-validation groups equals the number of samples, thus, only one sample is included in the validation group at a time [31]. When the cross-validation is run, the software provided an automatic measurement of these models' performance through their F1 score, a harmonic mean of the precision and recall of a binary classification system, i.e., a classification of positive and negative samples [32]. Here 'precision' is defined as the number of identified true positives among all the samples classified by the studied method as 'positives', and recall is the number of positive samples correctly identified as positive by the studied method within the total population of positive samples [33]. In other words, the closer the F1 score of a model is to 1 , the more accurate a classification model is. This is further outlined in the equation below:

$$
\mathrm{F}_{1}=\frac{2}{\text { recall }^{-1}+\text { precision }^{-1}}=2 \cdot \frac{\text { precision } \cdot \text { recall }}{\text { precision }+ \text { recall }}=\frac{\mathrm{TP}}{\mathrm{TP}+\frac{1}{2}(\mathrm{FP}+\mathrm{TP})}
$$

where ' $\mathrm{TP}^{\prime}$ ' is the number of true positives and ' $\mathrm{FP}^{\prime}$ ' is the number of false positives.

Once the models were created, their F1 score and predicted classification values were used to select the most suitable model(s) to be evaluated during the testing stage of the study.

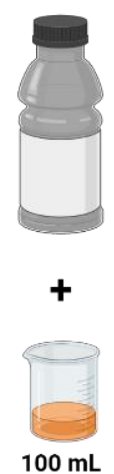

$100 \mathrm{~mL}$
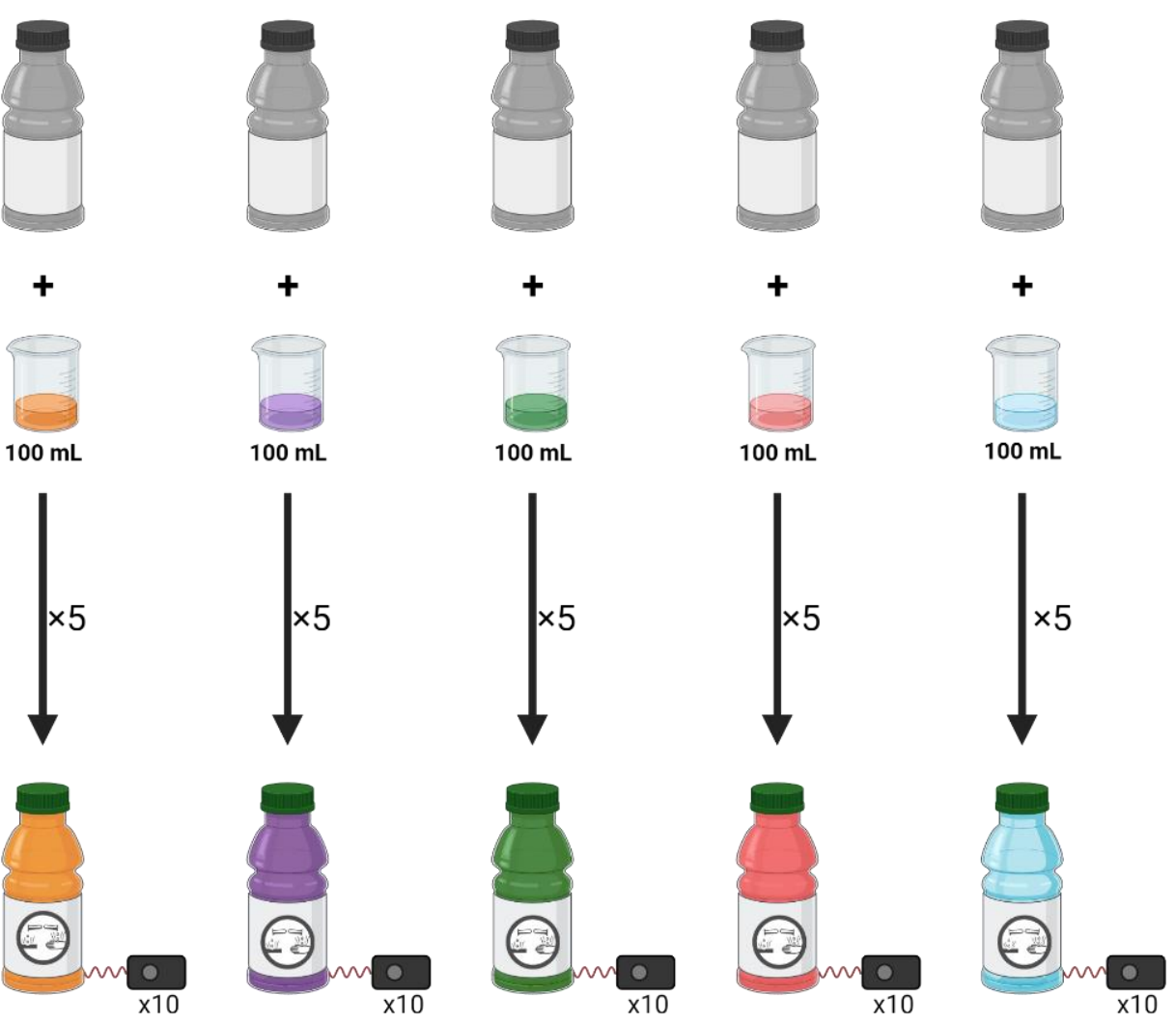

Figure 2. Graphical summary of the sample preparation and analysis during the data collection stage. Aliquots of $100 \mathrm{~mL}$ of each corrosive substance were poured into plastic bottles of PET and HDPE. Five aliquots of each combination of corrosive substance and plastic material were prepared and scanned 10 times each using NIR spectroscopy. Created with BioRender.com. 

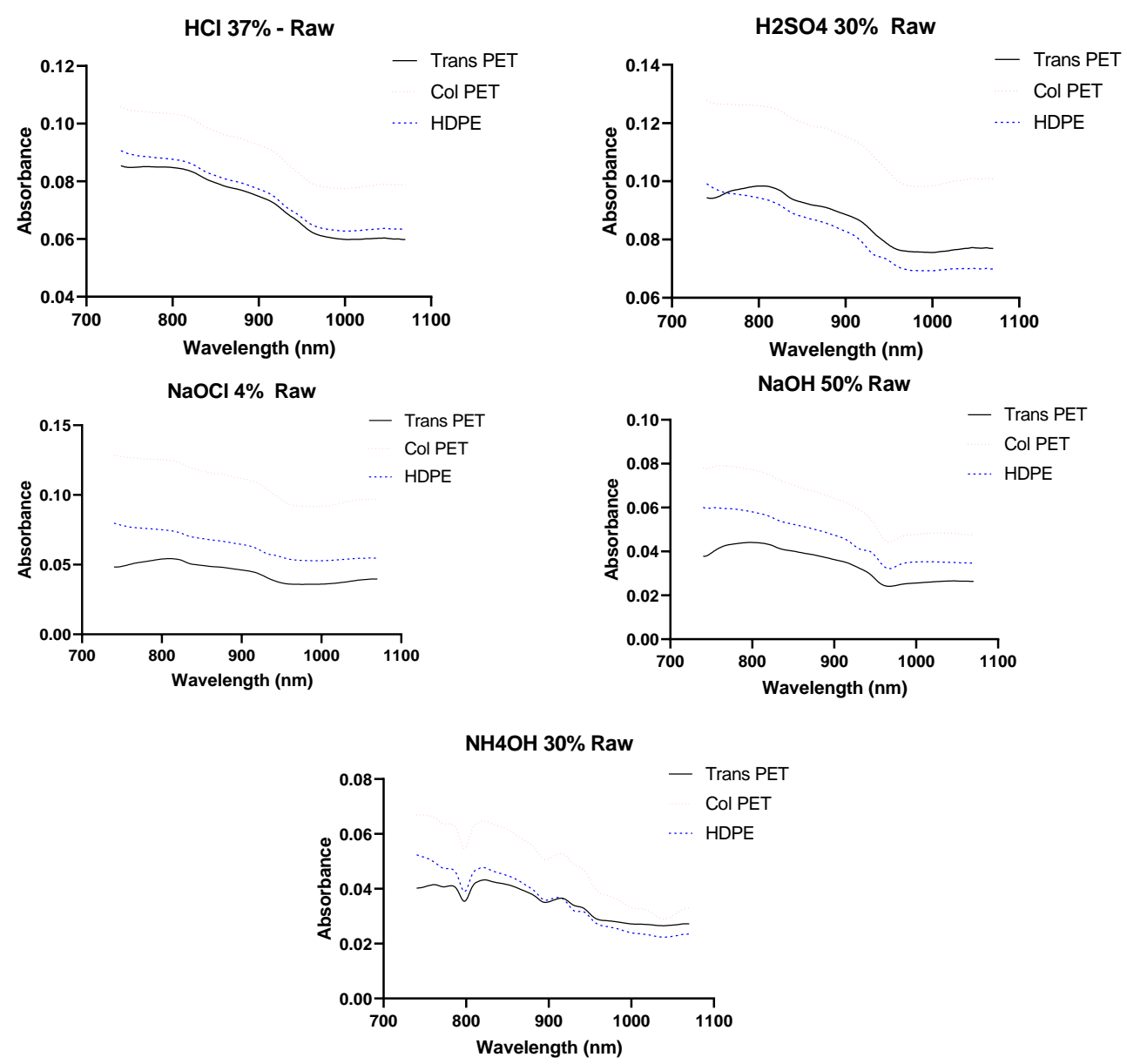

Figure 3. Examples of raw spectra of $\mathrm{HCl} 37 \%, \mathrm{H}_{2} \mathrm{SO}_{4} 30 \%, \mathrm{NaOCl} 4 \%, \mathrm{NaOH} 50 \%$ and $\mathrm{NH}_{4} \mathrm{OH}$ $30 \%$ scanned through bottles of Transparent PET (Trans PET), Coloured PET (Col PET) and HDPE. Raw spectra were downloaded using the $\mathrm{SCiO}^{\mathrm{TM}} \mathrm{Lab}$ application and then plotted using GraphPad Prism 8.0.1.

\subsection{Model Testing}

The model at this stage with the most optimal cross-validation performance that included all spectral data gathered during the training stage, hereon referred as "Model 1", was selected and tested against a new set of samples (i.e., 'testing set').

In a similar way to the data collection stage (Section 2.4), two sets of five samples of each corrosive, concentration and container were prepared in a new batch of plastic bottles. In addition to corrosive substances, two batches of negative samples (i.e., water, Lucozade and Fruit Shoot) were included into the training set to assess the performance of the created model(s) against safe substances. Empty bottles were also scanned as negative samples to evaluate the contribution plastic materials could have to the overall spectral signal. All the negative samples were prepared following the same protocol used for the positive samples, as described in Section 2.4.

Each sample in the testing set was scanned 10 times, making a total of 300 scans for each sample set, and the outcome, positive or negative, was noted to estimate the performance of a qualitative model. The output of each single scan was classified as True Positives (TP) if the corrosive sample was correctly classified, True Negatives (TN) if a 'safe' sample was not identified as a corrosive, False Positives (FP) if a 'safe' sample was identified as any of the corrosives and False Negatives (FN) when a corrosive sample was not classified as any of the defined classes, i.e., a 'null' result. In addition to this, some outputs were labelled as Misclassification (M) in situations where a corrosive sample was classified as a different corrosive. As the purpose of this study was to test the capability of NIR to discriminate between safe and corrosive substances, the relevance of these outputs 
is lower but nonetheless they are accounted for here. The substances that were not included in the library, such as water and soft drinks samples, were expected to provide 'null result', since no data was present within the training set to match their spectra with. For the purpose of these calculations, a null result is considered a negative result.

To further study and compare the performance of each of the final models tested, their 'Sensitivity' or 'True Positive Rate', often referred as a model's ability to correctly identify a true positive sample, were calculated following the equation below:

$$
\text { Sensitivity }=\frac{\mathrm{TP}}{\mathrm{TP}+\mathrm{FN}}
$$

\subsection{Model Optimisation}

After initial testing, additional models, Models 2, 3, and 4, were developed to further improve correct identification. These models were designed and tested using the same algorithms described in Section 2.5 but only included the training data from the concentrated aliquots for Model 2 or the diluted solutions for Models 3 and 4. In addition to this, Model 4 would use the average values from three scans before providing an output to determine whether this feature had any effect on its performance against new samples. The early capabilities of these models were estimated through internal cross-validation prior to be run against a new set of testing samples (prepared in the exact same manner as per Section 2.6). In addition to this, a McNemar's test was run on Models 3 and 4 to compare the number of TP and FN on MS Excel to assess the impact of multiple scans in the sensitivity of the model.

\section{Results}

At the training stage, which entails both data collection and data analysis, each corrosive was prepared in aliquots of at least two different concentrations, each tested in three types of plastic containers. In total, the training set included 480 samples, each scanned 10 times, with a total of 4800 scans in the created library.

Most of the samples prepared in this study were stable in the different plastic containers, albeit some slight alterations of the container, such as discoloration or deformation of the plastic $24 \mathrm{~h}$ after exposure to the corrosive substances. One exception was the case of sulfuric acid 97\%, which corroded the plastic material of the PET bottles in less than $20 \mathrm{~min}$. This highlights the fact that standard plastic bottles can hold highly dangerous substances without much risk to the assailant for most household products.

\subsection{Data Analysis}

Once the spectral library was constructed, the data was analysed in order to build chemometric models using a selection of available pre-processing methods. Initial models (Models from 0.1 to 0.4 ) were designed to evaluate the impact different pre-processing methods had on their performance during internal cross-validation.

The first model of this study, 'Model $0.1^{\prime}$, was based on spectra from all 480 samples within the library. This model produced cross-validation data that indicated the possibility of using this method for the qualitative discrimination of corrosive substances with an F1 score of 0.818 . During cross-validation there were, however, some samples that were misclassified, with the most evident cases being hydrochloric acid samples (where $8 \%$ of these were misclassified as sodium hypochlorite) and sulfuric acid (where $4 \%$ of the samples were assigned as hydrochloric acid). Since this model included samples of highly concentrated sulfuric acid and sodium hypochlorite prepared only in HDPE bottles, these were filtered out to avoid predictive instability due to an uneven sample distribution between the defined classes. Thus, 'Model 0.2' was created. The performance of Model 0.2 showed little difference compared to Model 0.1 , with an F1 score of 0.815 , actually indicating an overall slight decrease of performance during cross-validation. A closer inspection of the data revealed that the misclassification seen earlier for sulfuric acid had gone down to $0 \%$ and the percentage of samples of hydrochloric acid classified as sodium 
hypochlorite became $7 \%$. It was also observed that the regions below $800 \mathrm{~nm}$ and above $1000 \mathrm{~nm}$ of the raw spectra appeared to be noisy; these were, therefore, filtered out for all the other models, which included data only from the region between 800 and $1000 \mathrm{~nm}$. For the next model, 'Model 0.3', this specific wavelength region was used, along with data from all 480 samples. This model showed some classification improvement, with an F1 score of 0.889 and the previously observed misclassification decreasing to $5 \%$ for hydrochloric acid and 1\% for sulfuric acid. A final model refinement (Model 0.4) involved using the aforementioned wavelength region, as well as filtering out the samples from highly concentrated sulfuric acid and sodium hypochlorite in HDPE bottles. Model 0.4 appeared to have a lower F1 score (0.876) compared to Model 0.3, but the overall percentage of samples misclassified during cross-validation had greatly decreased, with $1 \%$ of samples misclassified in the case of hydrochloric acid and $0 \%$ for sulfuric acid. This final refined model — hereon referred as 'Model 1' —-was therefore selected for initial assessment against the Testing set.

\subsection{Model Testing}

Model 1 was tested against a new set of samples that included samples of corrosive substances contained in three types of bottles (refer to Section 2.4) as well as negative samples of soft-drinks, water and empty bottles (i.e., safe substances).

The results obtained are summarised in Tables 2 and 3.

Table 2. Classification of corrosive solutions scanned through the walls of transparent and coloured PET and HDPE bottles using Model 1. TP = True Positives, FN = False Negatives, $\mathrm{M}=$ Misclassification.

\begin{tabular}{cccc}
\hline Solution & TP & FN & M \\
\hline $\mathrm{HCl} 37 \%$ & 261 & 38 & 1 \\
$\mathrm{HCl} 10 \%$ & 198 & 95 & 7 \\
$\mathrm{NaOH} 50 \%$ & 286 & 14 & 0 \\
$\mathrm{NaOH} \mathrm{12 \%}$ & 229 & 71 & 0 \\
$\mathrm{NH}_{4} \mathrm{OH} \mathrm{30 \%}$ & 278 & 22 & 0 \\
$\mathrm{NH}_{4} \mathrm{OH} \mathrm{10 \%}$ & 195 & 103 & 2 \\
$\mathrm{H}_{2} \mathrm{SO}_{4} 30 \%$ & 268 & 32 & 0 \\
$\mathrm{H}_{2} \mathrm{SO}_{4} 15 \%$ & 226 & 74 & 0 \\
$\mathrm{NaHClO}_{3} 4 \%$ & 149 & 148 & 3 \\
$\mathrm{NaHClO}_{3} 1 \%$ & 200 & 100 & 0 \\
\hline
\end{tabular}

Table 3. Classification of safe substances scanned through the walls of PET and HDPE bottles using Model 1. TN = True Negatives and FP $=$ False Positives.

\begin{tabular}{ccc}
\hline Solution & TN & FP \\
\hline Empty & 300 & 0 \\
Water & 287 & 13 \\
Lucozade & 296 & 4 \\
Fruit shoot & 292 & 8 \\
\hline
\end{tabular}

Data gathered at this stage from the positive samples showed that Model 1 had a promising performance, with a high proportion of TP when testing the high concentration positive samples. The performance of the model appeared to slightly decrease as the concentration of the sample was reduced resulting in a small increase in FNs; however, no incidences of corrosive substances classified as a different corrosive were found. The performance was observed to remain unchanged across the three types of containers, indicating that the container material has minimal or no influence on the performance of the method. The only exceptions were hydrochloric acid and sodium hypochlorite. While the performance of Model 1 with concentrated samples of hydrochloric acid (37\%) was similar to that observed for sulfuric acid, sodium hydroxide and ammonium hydroxide, with diluted samples $(10 \%)$, the proportion of FN was somewhat higher than those observed with 
the concentrated solution. Moreover, samples of sodium hypochlorite proved challenging, with the proportion of FN being greater in the case of the concentrated samples than for the diluted ones, a behaviour that was not observed with the other corrosive substances at this stage. When studying the overall performance of this model, the sensitivity reached a value of 0.73 , in other words $73 \%$ of the positive samples were correctly identified using this model.

Samples of water, soft drinks, as well as empty plastic bottles (i.e., 'safe' samples), were scanned in a similar fashion as the positive samples to serve as negative controls and testing of Model 1 was carried out. Empty bottles gave no FP rather giving a 'null result' in all scans. This evidence showed that the plastic containers used in this study have a negligible effect on the overall spectra of the sample and confirmed one of the main benefits of NIR spectroscopy. In addition to this, the samples of soft drinks also proved successful, with only a small fraction of the samples-the highest incidence was 8 out of 100 samples-giving a FP result. These results provide further support to the capability of the developed model to correctly discriminate a safe substance from any of the five corrosives defined within the data library.

\subsection{Model Optimisation}

The results obtained so far showed that, despite Model 1 being capable of correctly identifying a high proportion of the concentrated positive samples, it achieved a lower performance against the diluted samples than the one observed when testing the model against the more concentrated aliquots. Thus, it was concluded that further improvement and optimization was required. The data within the training set was divided into two sets, one containing the spectra of the most concentrated corrosive samples and one with the spectra from the diluted samples. The reasoning behind this approach was to explore the influence of the presence or absence of data from concentrated and diluted samples on the performance of the model. At this stage, two models with different training libraries were designed and tested simultaneously to assess their performance against new samples. Model 2-concentrations above 15\%-and Model 3-concentrations below 15\%-were created and tested against a testing set identical to the one described in Section 3.2. In addition to these, a fourth model, Model 4, was designed using the data from those samples with a concentration below $15 \%$ as for Model 3, but, as an additional requirement, the model averaged the data from three scans before producing an output, (unlike prior models that used the data from a single scan) with the aim of increasing its overall accuracy. The results obtained from testing Models 2, 3, and 4 have been summarised in Tables 4-6, respectively.

Table 4. Classification of corrosive solutions scanned through the walls of transparent and coloured PET and HDPE bottles using Model 2. TP = True Positives, FN = False Negatives, $\mathrm{M}=$ Misclassification.

\begin{tabular}{cccc}
\hline Solution & TP & FN & M \\
\hline $\mathrm{HCl} 37 \%$ & 294 & 6 & 0 \\
$\mathrm{HCl} 10 \%$ & 142 & 97 & 61 \\
$\mathrm{NaOH} 50 \%$ & 291 & 9 & 0 \\
$\mathrm{NaOH} \mathrm{12 \%}$ & 142 & 118 & 40 \\
$\mathrm{NH}_{4} \mathrm{OH} \mathrm{30 \%}$ & 300 & 0 & 0 \\
$\mathrm{NH}_{4} \mathrm{OH} 10 \%$ & 96 & 104 & 100 \\
$\mathrm{H}_{2} \mathrm{SO}_{4} 30 \%$ & 294 & 6 & 0 \\
$\mathrm{H}_{2} \mathrm{SO}_{4} 15 \%$ & 297 & 3 & 0 \\
\hline
\end{tabular}

Model 2 showed a high proportion of TPs when used against the concentrated samples of all the tested corrosive solutions, with a rate of FN slightly lower than those observed with Model 1. However, when using Model 2 against diluted corrosive samples, which were not included in the dataset used to build this model, the performance appeared to be poorer compared to Model 1, with a higher rate of FN as well as of M, an occurrence that was barely present during the testing of Model 1 . Nevertheless, when studying the 
overall sensitivity of this model, the final value reached 0.84 , higher than that observed in Model 1 (0.73).

Table 5. Classification of corrosive solutions scanned through the walls of transparent and coloured PET and HDPE bottles using Model 3. TP = True Positives, FN = False Negatives, $\mathrm{M}=$ Misclassification . Sample sets marked with a '-' showed null results for all the samples scanned.

\begin{tabular}{cccc}
\hline Solution & TP & FN & M \\
\hline $\mathrm{HCl} 37 \%$ & - & - & - \\
$\mathrm{HCl} 10 \%$ & 229 & 70 & 1 \\
$\mathrm{NaOH} 50 \%$ & - & - & - \\
$\mathrm{NaOH} \mathrm{12 \%}$ & 292 & 8 & 0 \\
$\mathrm{NH}_{4} \mathrm{OH} 30 \%$ & - & - & - \\
$\mathrm{NH}_{4} \mathrm{OH} \mathrm{10 \%}$ & 300 & 0 & 0 \\
$\mathrm{H}_{2} \mathrm{SO}_{4} 30 \%$ & - & - & - \\
$\mathrm{H}_{2} \mathrm{SO}_{4} 15 \%$ & 214 & 69 & 16 \\
$\mathrm{NaHClO}_{3} 4 \%$ & 297 & 3 & 0 \\
$\mathrm{NaHClO}_{3} 1 \%$ & 292 & 7 & 1 \\
\hline
\end{tabular}

Table 6. Classification of corrosive solutions scanned through the walls of transparent and coloured PET and HDPE bottles using Model 4. TP = True Positives, FN = False Negatives, $\mathrm{M}=$ Misclassification. Sample sets marked with a '-' showed null results for all the samples scanned.

\begin{tabular}{cccc}
\hline Solution & TP & FN & M \\
\hline $\mathrm{HCl} 37 \%$ & - & - & - \\
$\mathrm{HCl} 10 \%$ & 279 & 21 & 0 \\
$\mathrm{NaOH} 50 \%$ & - & - & - \\
$\mathrm{NaOH} 12 \%$ & 299 & 1 & 0 \\
$\mathrm{NH}_{4} \mathrm{OH} 30 \%$ & - & - & - \\
$\mathrm{NH}_{4} \mathrm{OH} 10 \%$ & 300 & 0 & 0 \\
$\mathrm{H}_{2} \mathrm{SO}_{4} 30 \%$ & - & - & - \\
$\mathrm{H}_{2} \mathrm{SO}_{4} 15 \%$ & 253 & 38 & 8 \\
$\mathrm{NaHClO}_{3} 4 \%$ & 300 & 0 & 0 \\
$\mathrm{NaHClO}_{3} 1 \%$ & 300 & 0 & 0 \\
\hline
\end{tabular}

On the other hand, Model 3 appeared to be able to correctly classify diluted samples of all corrosive solutions in most cases, with the highest rate of FN and FP seen with diluted samples of sulfuric acid., The model was also able to correctly classify highly diluted samples, (i.e., sodium hypochlorite $1 \%$ ). However, the model was unable to classify most concentrated samples of corrosive solutions, in most cases giving an 'Invalid' output. Model 4, which used the same dataset as Model 3 but with three averaged scans, showcased a similar performance to Model 3 but with a higher rate of TP when analysing the diluted samples, nearing a perfect classification in cases such as $\mathrm{NH}_{4} \mathrm{OH} 10 \%$. Due to the high proportion of samples with an invalid output, the sensitivity observed for Model 3 and 4 were 0.54 and 0.58 , lower than those gathered from Models 1 and 2. Nonetheless, the overall performances for both model 3 and 4, appear not as different (refer to Figure 4). By performing a McNemar's test to compare the number of TP of these two models, a $X^{2}$ value of 3.35 and a $p$-value of 0.07 are observed. This shows that the null hypothesisboth models show no statistical difference in terms of the number of true positives-can be accepted, thus further confirming that there is no statistical difference between using single-scan and triple-scan analysis.

As it is to be expected, a model built with a specific selection of concentrations is capable to perform within a favourable range when tested against samples of the same concentrations as those included within the training set. 


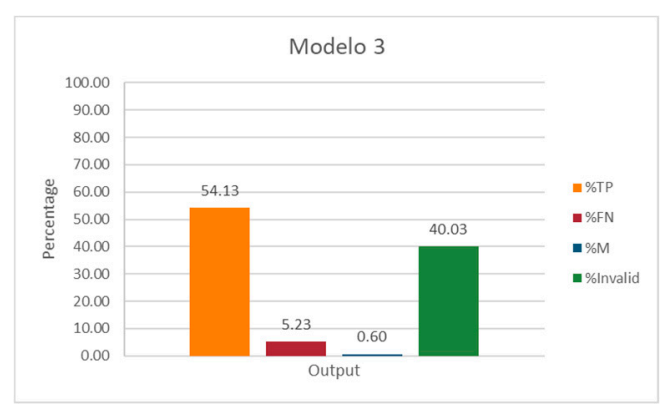

(a)

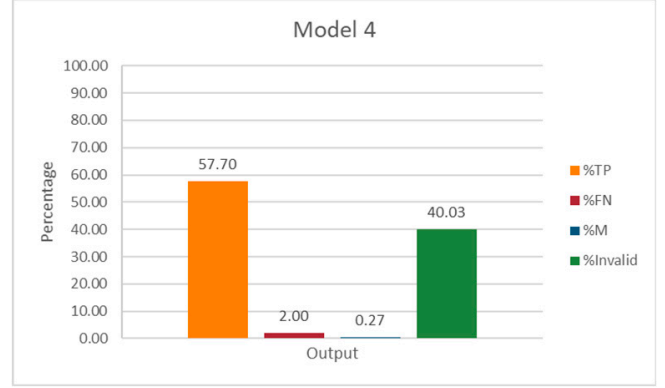

(b)

Figure 4. Percentage of total True Positives (\%TP), False Negatives (\%FN), Misclassifications (\%M) and 'Invalid' output, for (a) Model 3, using single scan data, and (b) Model 4, using triple scan data.

\section{Conclusions}

This study has explored the capability of portable NIR spectroscopy for the identification of inorganic acids and bases through plastic containers. Although designed as an early feasibility study, the work outlined here has shown that, within laboratory conditions, near infrared is able to identify inorganic corrosive substances through closed containers with accuracy. The results have also proven that a chemometric model, such as Model 1, designed with the tools available and the data gathered with this commercial instrument, can discriminate a safe substance, such as water, from a corrosive solution, whilst not being affected by the container itself.

On the other hand, the results have demonstrated how creating a varied and large dataset is important in the design of a chemometric model. Future work in this line of research could include the design of a spectral library that covers a range of solutions and concentrations resembling nearly all potential real case scenarios. This could then be tested against a new set of samples to determine whether a single model can be used to discriminate all potential concentrations. Furthermore, the samples prepared in this study were aqueous solutions of analytical grade inorganic acids and bases that did not include any additional component that may be present in the household products, which are commonly used in incidents involving corrosive substances. At the present, the current literature lacks detailed information on the analysis of inorganic acid and bases using Near Infrared spectroscopy. The source of misclassification observed in this study may be related to the signals that these different molecules, or their interaction with water molecules, may or may not present within the detected region. A better understanding of the spectral signature of these corrosive molecules would provide great insights on their influence in the NIR spectral, which in turn would aid in the improvement of the fitness of this method. Future method validation could include a new testing set comprised of commercially available solutions to assess whether these supplementary compounds can affect the analysis in a real-life case scenario. Once these aspects have been studied and the method is fully validated, it is expected that this technique could prove useful in the fight against crimes involving concealed corrosive substances and constitute a great advantage for police forces when analysing hazardous substances on the field.

Author Contributions: Conceptualization, methodology, resources, investigation, validation, visualization and writing—original draft by A.V.M. Supervision and writing—review and editing by N.F. All authors have read and agreed to the published version of the manuscript.

Funding: This research received no external funding.

Data Availability Statement: The data presented in this study is openly available in https:/ / kcl. figshare.com/ at 10.18742/19316639.

Acknowledgments: The authors would like to thank Dan Wood and Richard Warren from the Threat Mitigation Technologies team at the Metropolitan Police Service for their guidance as well as for supporting this research through the donation of relevant materials and reagents used in the study. 
Conflicts of Interest: The authors declare no conflict of interest.

\section{References}

1. Hopkins, M.; Neville, L.; Sanders, T. The Motivations of Offenders Who Carry and Use Acid and Other Corrosives in Criminal Acts; University of Leicester: Leicester, UK, 2021; pp. 1-94.

2. Stripe, N. Crime in England and Wales Year Ending September 2020; Office for National Statistics: London, UK, 2021.

3. Reich, G. Near-infrared spectroscopy and imaging: Basic principles and pharmaceutical applications. Adv. Drug Deliv. Rev. 2005, 57, 1109-1143. [CrossRef] [PubMed]

4. Bai, S.; Nayar, R.; Carpenter, J.F.; Manning, M.C. Noninvasive determination of protein conformation in the solid state using near infrared (NIR) spectroscopy. J. Pharm. Sci. 2005, 94, 2030-2038. [CrossRef] [PubMed]

5. Cozzolino, D.; Kwiatkowski, M.J.; Waters, E.J.; Gishen, M. A feasibility study on the use of visible and short wavelengths in the near-infrared region for the non-destructive measurement of wine composition. Anal. Bioanal. Chem. 2007, 387, 2289-2295. [CrossRef] [PubMed]

6. Hammond, J.; Moffat, A.C.; Jee, R.D.; Kellam, B. Non-destructive real-time reaction monitoring in solid phase synthesis by near-infrared reflectance spectroscopy-Esterification of a resin-bound alcohol. Anal. Commun. 1999, 36, 127-129. [CrossRef]

7. Kamat, M.S.; Lodder, R.A.; DeLuca, P.P. Near-Infrared Spectroscopic Determination of Residual Moisture in Lyophilized Sucrose Through Intact Glass Vials. Pharm. Res. 1989, 6, 961-965. [CrossRef]

8. Morillas, A.V.; Gooch, J.; Frascione, N. Feasibility of a handheld near infrared device for the qualitative analysis of bloodstains. Talanta 2018, 184, 1-6. [CrossRef]

9. $\quad$ Pereira, J.F.Q.; Silva, C.S.; Vieira, M.J.L.; Pimentel, M.F.; Braz, A.; Honorato, R.S. Evaluation and identification of blood stains with handheld NIR spectrometer. Microchem. J. 2017, 133, 561-566. [CrossRef]

10. Sharififar, A.; Singh, K.; Jones, E.; Ginting, F.I.; Minasny, B.; Aitkenhead, M. Evaluating a low-cost portable NIR spectrometer for the prediction of soil organic and total carbon using different calibration models. Soil Use Manag. 2019, 35, 607-616. [CrossRef]

11. Sun, Y.; Wang, Y.; Huang, J.; Ren, G.; Ning, J.; Deng, W.; Li, L.; Zhang, Z. Quality assessment of instant green tea using portable NIR spectrometer. Spectrochim. Acta A Mol. Biomol. Spectrosc. 2020, 240, 118576. [CrossRef]

12. Edelman, G.; Manti, V.; van Ruth, S.M.; van Leeuwen, T.; Aalders, M. Identification and age estimation of blood stains on colored backgrounds by near infrared spectroscopy. Forensic Sci. Int. 2012, 220, 239-244. [CrossRef]

13. Candolfi, A.; Wu, W.; Massart, D.L.; Heuerding, S. Comparison of classification approaches applied to NIR-spectra of clinical study lots. J. Pharm. Biomed. Anal. 1998, 16, 1329-1347. [CrossRef]

14. Sun, J. Statistical analysis of NIR data: Data pretreatment. J. Chemom. 1997, 11, 525-532. [CrossRef]

15. Barnes, R.J.; Dhanoa, M.S.; Lister, S.J. Standard Normal Variate Transformation and De-Trending of Near-Infrared Diffuse Reflectance Spectra. Appl. Spectrosc. 1989, 43, 772-777. [CrossRef]

16. Abdi, H.; Williams, L.J. Partial least squares methods: Partial least squares correlation and partial least square regression. In Computational Toxicology: Volume II; Reisfeld, B., Mayeno, A.N., Eds.; Humana Press: Totowa, NJ, USA, 2013 ; pp. 549-579.

17. Wold, S.; Sjöström, M. SIMCA: A Method for analyzing chemical data in terms of similarity and analog. In Chemometrics: Theory and Application; American Chemical Society: Columbus, OH, USA, 1977; pp. 243-282.

18. Krämer, K.; Ebel, S. Application of NIR reflectance spectroscopy for the identification of pharmaceutical excipients. Anal. Chim. Acta 2000, 420, 155-161. [CrossRef]

19. Chalus, P.; Roggo, Y.; Walter, S.; Ulmschneider, M. Near-infrared determination of active substance content in intact low-dosage tablets. Talanta 2005, 66, 1294-1302. [CrossRef]

20. Risoluti, R.; Materazzi, S.; Gregori, A.; Ripani, L. Early detection of emerging street drugs by near infrared spectroscopy and chemometrics. Talanta 2016, 153, 407-413. [CrossRef]

21. Udelhoven, T.; Emmerling, C.; Jarmer, T. Quantitative analysis of soil chemical properties with diffuse reflectance spectrometry and partial least-square regression: A feasibility study. Plant Soil 2003, 251, 319-329. [CrossRef]

22. Ulmschneider, M.; Wunenburger, A.; Pénigault, E. Using Near-Infrared Spectroscopy for the noninvasive identification of five pharmaceutical active substances in sealed vials. Analusis 1999, 27, 854-856. [CrossRef]

23. Barri, K.; Jahangiri, B.; Davami, O.; Buttlar, W.G.; Alavi, A.H. Smartphone-based molecular sensing for advanced characterization of asphalt concrete materials. Measurement 2020, 151, 107212. [CrossRef]

24. Bickler, M.P.; Rhodes, L.J. Accuracy of detection of carboxyhemoglobin and methemoglobin in human and bovine blood with an inexpensive, pocket-size infrared scanner. PLOS ONE 2018, 13, e0193891. [CrossRef]

25. Li, M.; Qian, Z.; East, A.R. Does consumer-scale near-infrared (NIR) spectroscopy provide opportunities for kiwifruit quality measurement? Acta Hortic. 2018, 1218, 481-488. [CrossRef]

26. Li, M.; Qian, Z.; Shi, B.; Medlicott, J.; East, A. Evaluating the performance of a consumer scale SCiO ${ }^{\mathrm{TM}}$ molecular sensor to predict quality of horticultural products. Postharvest Biol. Technol. 2018, 145, 183-192. [CrossRef]

27. Pastor, R.F.; Mariani, M.L.; Villach, M.G.; Cascón, P.N.; Giudice, M.; Pastor, E.; Pastor, I.; Iermoli, R.H.; Penissi, A.B. A Novel Approach to Measure the Total Antioxidant Power of Wines through Near Infrared Spectroscopy and Its Relevance in Human Nutrition. J. Health Sci. 2019, 7, 209-214.

28. Westad, F.; Marini, F. Validation of chemometric models-A tutorial. Anal. Chim. Acta 2015, 893, 14-24. [CrossRef]

29. Geladi, P.; Kowalski, B.R. Partial least-squares regression: A tutorial. Anal. Chim. Acta 1986, 185, 1-17. [CrossRef] 
30. Haenlein, M.; Kaplan, A.M. A Beginner's Guide to Partial Least Squares Analysis. Underst. Stat. 2004, 3, 283-297. [CrossRef]

31. Ballabio, D.; Consonni, V. Classification tools in chemistry. Part 1: Linear models. PLS-DA. Anal. Methods 2013, 5, 3790-3798. [CrossRef]

32. Sasaki, Y. The Truth of the F-Measure; University of Manchester: Manchester, UK, 2007; p. 5.

33. Powers, D. Evaluation: From Precision, Recall and F-Factor to ROC, Informedness, Markedness \& Correlation. Int. J. Mach. 2011, 2, 37-63. 